\title{
HOW TO EVALUATE QUALITY FOR THORACIC LUNG CANCER SURGERY: A 'TEXTBOOK OUTCOME"'
}

\author{
R. C. Numan ${ }^{a}$, P. Baas ${ }^{b}$, J. A. Burgers ${ }^{b}$, V. vd Noort ${ }^{c}$, H. v Tinteren $^{c}$, J.W.
} $v$ Sandick $^{a}$, H. M. Klomp ${ }^{a}$ and M. W. Wouters ${ }^{a}$.

a Netherlands Cancer Institute/Antoni van Leeuwenhoek Hospital, Plesmanlaan 121, 1066CX Amsterdam. The Netherlands. Department of Surgical Oncology. j.v.sandick@nki.nl; h.klomp@nki.nl; m.wouters@nki.nl

${ }^{\mathrm{b}}$ Netherlands Cancer Institute/Antoni van Leeuwenhoek Hospital, Plesmanlaan 121, 1066CX Amsterdam. The Netherlands. Department of Thoracic Oncology.

p.baas@nki.nl; s.burgers@nki.nl

\begin{abstract}
${ }^{\mathrm{C}}$ Netherlands Cancer Institute/Antoni van Leeuwenhoek Hospital, Plesmanlaan 121, 1066CX Amsterdam. The Netherlands. Department of Statistics.

v. vd.noort@nki.nl; h.v.tinteren@nki.nl
\end{abstract}

\section{Corresponding author:}

Rachel C Numan

Netherlands Cancer Institute-Antoni van Leeuwenhoek Hospital. Department of

Surgical Oncology

Plesmanlaan 1211066 CX Amsterdam

Tel: 00-31-652579848 Fax : 00-31-205122459. email: r.numan@nki.nl

\section{Abstract}

Objectives-Measuring surgical quality gives rise to an ongoing debate on which quality of care indicators should be used. Individual measures such as postoperative mortality, do not fully reflect quality of care. Instead, a summarizing measure (i.e. a "textbook outcome") can be used. The objectives of this study were to investigate the proportion of patients with a "textbook outcome" and to identify variables reducing the chance for a "textbook outcome".

Materials and Methods-From January 2003 to December 2011, 152 patients operated for non-small cell lung carcinoma (NSCLC) were identified using both a prospective database $(\mathrm{N}=93)$ which was incorporated in a multidisciplinary care path for thoracic surgery in the Netherlands Cancer Institute, and a retrospective database $(\mathrm{N}=59)$. Patient-, tumour-, treatment- and outcome characteristics were collected. A "textbook outcome" was defined as a postoperative course without in-hospital death, without complications within 30 days, without re-intervention within 30 days, and without re-admission within 30 days after discharge, in combination with a radical tumour resection, a hospital stay $<16$ days and an Intensive Care stay $<3$ days.

Results - In 96 of 152 patients (63\%), a "textbook outcome" was realized. A logistic regression analysis including stage of disease, pulmonary co-morbidity, smoking 
status, lung function and type of resection showed that stage of disease was an independent risk factor that reduced the chance of a "textbook outcome"(OR 0.56 , $95 \% \mathrm{Cl}$ 0.33-0.92, $p=0.024)$.

Conclusions - A "textbook outcome" is a comprehensive, summarizing measure that has the potential to overcome limitations of individual measures describing postoperative outcome. This is the first study exploring the use of a "textbook outcome" for lung cancer surgery. We showed, in two thirds of the patients, a "textbook outcome" was realized, where only the stage of disease was identified as an independent negative risk factor.

Keywords - Quality of Care, lung cancer, surgery, textbook outcome, outcome indicator, quality improvement 


\section{Introduction}

Nowadays, measuring quality of care has become an essential element in delivering health care. Internationally, the European Society for Thoracic surgeons (ESTS) started measuring quality of care in 2001, making it possible to compare individual hospitals and improve quality of care [22]. In the Netherlands, the Dutch Institute for Clinical Auditing (DICA) started evaluating quality of care of surgery for colorectal cancer in 2009. Currently, they embedded 15 clinical audits in the Dutch health care system [21].

Measuring surgical quality gives rise to an ongoing debate on which quality of care indicators should be used. Structure, process and outcome indicators all have their specific qualities depending on the surgical field they are used for [2]. For thoracic lung cancer surgery, there is contradicting evidence about the effect of structure indicators such as hospital volume and surgeon specialty, on postoperative mortality $[18,14,13]$. Also, the effect of these structure indicators on nonfatal outcome parameters is not clear. There is more knowledge on process indicators in this surgical field, such as the use of PET-CT as staging method and the safe use of video-assisted thoracoscopic surgery (VATS) in early stage Non Small Cell Lung Cancer (NSCLC) [5, 6, 10]. Outcome indicators are the most commonly used quality of care measures in this field. They are easy to collect and directly reflect patients outcome. Outcome indicators also have disadvantages, especially for NSCLC surgery. In the Netherlands, 11,669 new cases of NSCLC were diagnosed in 2011, being the fourth most common cancer type, but only 10 percent was eligible for operation [20-
19]. Because of the low operability rates, thoracic surgery for lung cancer has a relatively low caseload per hospital per year with low mortality and morbidity rates [21]. It is well known that low event rates combined with small sample sizes limit statistical power, which could lead to problems identifying hospitals that perform below average [7].

It is difficult to compare hospitals on individual outcome indicators, such as mortality or morbidity: a hospital may perform above average on one quality of care indicator and below average on another, complicating hospital to hospital comparisons.

Therefore, alternative approaches to measure the quality of surgical care have been considered. A summarizing measure could give an overall view on quality of care, not only for health care providers and policy makers but also for patients. Dijs-Elsinga et al. showed that patients would use information about quality of care more easily when it is handed as a summarizing measure such as a "textbook outcome" [9]. This summary measure represents a quality of care measure in which all preferred health outcomes are incorporated, which makes a "textbook outcome" a desired quality measure for patients, medical professionals and policy makers.

The objectives of this study were to investigate the proportion of patients with a "textbook outcome" in a referral center for thoracic lung cancer surgery and to identify variables influencing the chance for a "textbook outcome".

\section{Material and Methods}

\subsection{Patients}

All patients operated for NSCLC by thoracotomy or VATS between April 1, 
2006 and December 31, 2011 were identified using a prospective database that was incorporated in a multidisciplinary care path for thoracic surgery in the Netherlands Cancer Institute [16]. All patients with (potentially) malignant pulmonary lesions scheduled for lung resection were asked to participate in this care path, which included a prospective data registration and was approved by the Institutional Review Board. Exclusion criteria for participation were age younger than 18 years, inability or refusal to complete quality of life questionnaires, and a lung resection being part of a chest wall resection. All patients included in the clinical care path gave written informed consent.

For this study, all patients who were operated for NSCLC and did not show tumour invasion in adjacent structures during operation were selected from the prospective database. Patients receiving preoperative treatment (i.e. chemotherapy, radiotherapy or both) or needing additional non-lung parenchymal resection, were excluded (Figure 1). Patient characteristics, including age, gender, smoking status, World Health Organisation (WHO) performance status and history of previous thoracic surgery, were documented. Pre-treatment evaluation included medical history, physical examination, diagnostic imaging and histological diagnosis. Details of the surgical intervention and anesthesiological proceedings were collected. Direct postoperative outcome parameters regarding postoperative morbidity and mortality, length of hospital stay, readmissions, chest tube duration, epidural duration and detailed pathology data were collected during hospital stay. QoL was evaluated through the short-form SF-36, an easy to use and validated questionnaire which assesses eight dimensions of perceived well-being and gives two composite measures: a physical composite scale (PCS) and mental composite scale (MCS) [15]. The four physical scales are physical functioning (PF), role-physical functioning (RP), bodily pain (BP) and general health $(\mathrm{GH})$; the four mental scales are social functioning (SF), role-emotional functioning (RE), vitality (VT) and mental health (MH). The scores for all eight dimensions range from 0 to 100 , with higher scores representing better health status. The SF-36 questionnaires were administered prior to the operation and 1, 3 and 6 months after the operation. The summary scales for overall physical and mental health-related QoL uses norm-based methods with higher scores indicating better health status. 


\section{Figure 1. Flow chart of inclusion and exclusion criteria}

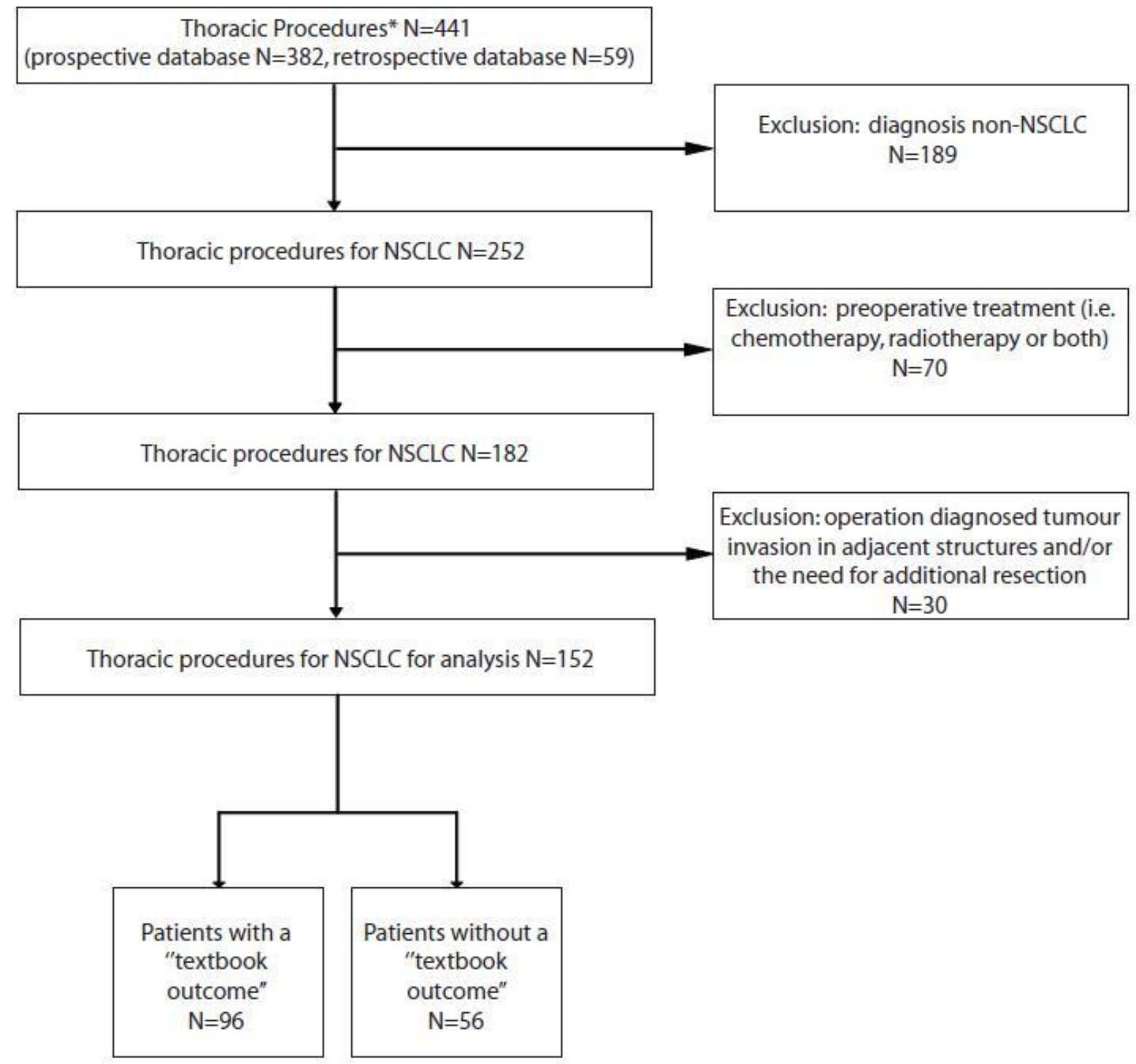

\section{Legend}

*The thoracic procedures consisted of 120 video assisted thoracoscopic procedures and 321 thoracotomies 
Data extracted from the prospective database was supplemented with data from a retrospective database containing all patients undergoing pulmonary resection for NSCLC between January 2003 and April 2006 in the Netherlands Cancer Institute. Patient, tumour, and outcome characteristics were retrieved from individual patient files, and were similar to those collected in the prospective database. Details of the surgical intervention and anesthesiological proceedings were collected. Using data from both the prospective and retrospective database, data on 152 patients were available for analysis.

\subsection{Surgery}

Eligible patients were planned for surgery using an open approach (i.e. thoracotomy) or a minimally invasive approach (i.e. VATS). When a thoracotomy was performed, the thoracic cavity was entered through the $5^{\text {th }}$ intercostal space. Evidence for the advances of a muscle sparing thoracotomy, led to the implementation of this operation technique in 2006 [16,17]. When a minimal invasive approach was used, two trocars were placed near the target location without spreading the ribs. Type of surgical resection depended on tumour size: wedge resection was the preferred surgical resection for small lesions, larger or centrally located tumours were resected by an anatomical segmentectomy or lobectomy. After surgical resection a chest tube was placed.

\subsection{Definitions}

The summarising "textbook outcome" measure consisted of seven "preferred outcome measures". These included being alive at discharge (i.e. no in-hospital death), no complications within 30 days after the operation, no re-intervention within 30 days after the operation, no re-admission within 30 days after discharge, a radical tumour resection (defined as a ccomplete resection with no microscopic residual tumor, in line with the "R" classification adopted by the Union for International Cancer Control in 1987), a hospital stay of less than 16 days, (mean hospital stay plus one standard deviation), and an Intensive Care stay of less than 3 days (mean intensive care stay plus one standard deviation).

Complications were included when clinically relevant (i.e. when medical intervention was needed). Lung related complications included: atelectasis for which bronchoscopic interference was needed, bleeding of the operation area, torsion and bronchoesophageal fistula. Also, pneumonia, empyema, wound infection requiring antibiotic treatment and prolonged air leakage $>7$ days for which drain insertion was needed, were scored as lung related complications. Non-lung related complications included arrhythmia, heart failure, sepsis/ARDS, kidney failure, myocard infarction and thrombosis, in which all conditions needed pharmacological interference or mechanical support. Deterioration of pre-existing conditions (such as preexistent cardiomyopathy with limited cardiac function) due to the operation ,were also scored as a complication. If data on one of the seven preferred outcome measures was missing, we marked the patient as having "no textbook outcome". An additional subanalysis was performed, supplementing the summarizing "textbook outcome" measure with quality of life data. In 72 patients complete quality of life questionnaires 6 months after the operation were retrieved. Because 3 and 6 months 
postoperative QoL data were comparable, in case of missing data concerning QoL 6 months after surgery, the QoL score 3 months after surgery was used. The percentage of patients with a PCS and MCS equal or above baseline six months after the operation, was included in the "textbook outcome".

\subsection{Risk factors}

Risk factors influencing the chance of a "textbook outcome" in thoracic lung cancer surgery have not been described in literature. Expert opinion (i.e. thoracic surgeons, lung physicians) based factors were included in the multivariate analysis: age, sex, WHO performance, body mass index (BMI), co-morbidity, smoking status, lung function (forced expiratory volume in one second and Lung Diffusing Capacity for Carbon monoxide), type of surgical incision, type of resection, muscle sparing technique and stage of disease.

\subsection{Statistical Analysis}

Differences between patients with a "textbook outcome" and those without were assessed by the chi squared or Fisher exact test for categorical variables and by a Student t-Test or Mann-Whitney $U$ test for continuous variables. To explore which variables reduced the chance of a "textbook outcome", a logistic regression analysis was performed. All expert based risk factors were entered in a multivariate model. Step by step excluding risk factors with $p>0.3$, the model identified independent predictors of a reduced chance of a "textbook outcome". A $p$ value of $<0.05$ was regarded statistically significant. All statistical analyses were performed in SPSS 20.0.0.

\section{Results}

From 2003 to 2011, a total of 441 thoracic procedures for (potentially) malignant pulmonary lesions were performed. To compile a homogeneous patient group, only patients with NSCLC were included $(\mathrm{N}=252)$. Patients receiving preoperative treatment (i.e. chemotherapy, radiotherapy or both) $(\mathrm{N}=70)$, patients with tumour invasion in adjacent structures during operation and patients needing additional non lung parenchymal resection $(\mathrm{N}=30)$, were excluded (Figure 1). For all 152 patients, data on the seven "preferred outcome measures" were complete.

Patient and tumour characteristics are shown in Table 1 . In 96 patients (63\%) a "textbook outcome" was observed. A hospital stay without complications was met in 72 percent of patients, making "complications" the indicator with the highest event rate (Table 2). Differences in baseline characteristics were seen between patients with and those without a "textbook outcome". Less disease burden (i.e. early stage NSCLC, $\quad p<0.0001)$ and better pulmonary function $(p=0.030)$ were seen in patients with a "textbook outcome". Also, patients with a "textbook outcome", showed less severe smoking addiction (i.e. less pack years, $p=0.045$ ) compared to patients without a "textbook outcome". Finally, faster removal of the epidural catheter and chest tube was observed in patients with a "textbook outcome" (both $p<0.0001)$ (Table 1). 
Table 1. Patient, tumour treatment and outcome characteristics of patients with and without a 'textbook outcome'

\begin{tabular}{|c|c|c|c|}
\hline Characteristics & $\begin{array}{c}\text { Patients with a } \\
\text { textbook } \\
\text { outcome } N=96\end{array}$ & $\begin{array}{c}\text { Patient without a } \\
\text { textbook } \\
\text { outcome } \mathrm{N}=56\end{array}$ & $P$ value \\
\hline Age, yrs (Mean, Range) & $68 \pm 10.9$ & $69 \pm 9.3$ & 0.584 \\
\hline \multicolumn{4}{|l|}{ Gender (No., \%) } \\
\hline $\mathrm{M}: \mathrm{F}$ & $42: 54(43.8: 56.3)$ & 25:31 (44.6: 55.4) & 0.915 \\
\hline \multicolumn{4}{|l|}{ Smoking status (No., \%) } \\
\hline Never & $6(6.3)$ & $1(1.8)$ & 0.526 \\
\hline Former & $58(60.4)$ & $36(64.3)$ & \\
\hline Current & $31(32.3)$ & 19 (33.9) & \\
\hline Unknown & $1(1.0)$ & $0(0)$ & \\
\hline Pack Years (median, SD) & $32 \pm 19.1$ & $40 \pm 21.8$ & 0.118 \\
\hline BMI (mean, SD) & $26 \pm 4.9$ & $25 \pm 3.9$ & 0.534 \\
\hline \multicolumn{4}{|l|}{ Co-morbidity } \\
\hline Pulmonary & $23(24.0)$ & $22(39.3)$ & 0.046 \\
\hline Cardiac & $15(15.6)$ & $8(14.3)$ & 0.826 \\
\hline Diabetes Mellitus & $9(9.4)$ & $6(10.7)$ & 0.789 \\
\hline Vascular & $29(30.2)$ & $17(30.4)$ & 0.985 \\
\hline \multicolumn{4}{|l|}{ WHO-performance } \\
\hline $0: 1: 11$ & $\begin{array}{c}60(62.5): 36(37.5): \\
0(0)\end{array}$ & $\begin{array}{c}29(51.8): 26(46.4): \\
1(1.8)\end{array}$ & 0.212 \\
\hline Pre-operative FEV1 ${ }^{a}$ & $89 \pm 17.6$ & $82 \pm 18.6$ & 0.026 \\
\hline $\begin{array}{l}\text { Pre-operative TLCo } \\
\text { (\% of expected, IQ range) }\end{array}$ & $77 \pm 18.2$ & $73 \pm 20.4$ & 0.282 \\
\hline \multicolumn{4}{|l|}{$\begin{array}{l}\text { Surgical technique (No. } \\
\text { of patients, \%) }\end{array}$} \\
\hline $\begin{array}{l}\text { Thoracotomy } \\
\text { VATS }\end{array}$ & $\begin{aligned} 90 & (93.8) \\
6 & (6.2)\end{aligned}$ & $\begin{array}{c}54(96.4) \\
2(3.6)\end{array}$ & 0.711 \\
\hline \multicolumn{4}{|l|}{$\begin{array}{l}\text { Resections (No. of } \\
\text { patients, \%) }\end{array}$} \\
\hline $\begin{array}{l}\text { Wedge resection } \\
\text { Segmentectomy }\end{array}$ & $\begin{aligned} 8 & (8.3) \\
25 & (26.0)\end{aligned}$ & $\begin{array}{c}3(5.4) \\
8(14.3)\end{array}$ & 0.239 \\
\hline
\end{tabular}




Lobectomy
Bilobectomy
Sleeve resection
Pneumonectomy
Muscle sparing
Yes
No
Thoracic drainage days
(Mean \pm SD)

Epidural analgesia days
(Mean $\pm S D$ )

Tumour stage (No. of
patients, \%)
Stage 0 (in situ)
Stage 1a
Stage 1b
Stage 2a
Stage $2 b$
Stage 3a
Stage 3b

Adjuvant Therapy (No. of patients, \%)

No therapy

Chemotherapy

Radiotherapy

Chemoradiation

Survival (alive at 201411-01)

(No. of patients, \%)

Yes

No

Unknown

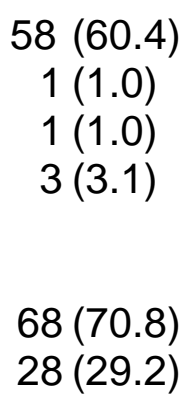

$58(60.4)$
$1(1.0)$
$1(1.0)$
$3(3.1)$

$68(70.8)$
$28(29.2)$

$5 \pm 2.1$

$3(3.1)$

$51(53.1)$

22 (22.9)

$3(3.1)$

$7(7.3)$

$10(10.4)$

$0(0)$

$1(1.8)$

$37(66.1)$

$3(5.4)$

$1(1.8)$

$4(7.1)$

$33(58.9)$

$23(41.1)$

0.134

$10 \pm 8.2$

$<0.0001$

$11(29.6)$

17 (30.4)

5 (8.9)

$15(26.8)$

$5(8.9)$

2 (3.6)

$<0.0001$

0.304

$38(67.9)$

9 (16.1)

$5(8.9)$

$4(7.1)$

$<0.0001$

$12(12.5)$

4 (4.2)

$3(3.1)$

0.421

\section{Legend}

$a_{\text {FEV1 Forced expiratory volume in one second }}$

${ }^{b}$ TLCO The carbon monoxide transfer factor 
Table 2. Outcome after pulmonary resection for NSCLC

\begin{tabular}{lcc}
\hline & \multicolumn{2}{c}{ Patients } \\
\cline { 2 - 3 } & $\mathrm{N}$ & $\%$ \\
\hline Total patients included & 152 & 100 \\
& & \\
\hline No in hospital Mortality & 148 & 97.4 \\
\hline No readmission & 144 & 94.7 \\
\hline Intensive Care stay<3 days & 143 & 94.1 \\
\hline Radical Resection & 140 & 92.1 \\
\hline No re-intervention & 139 & 91.4 \\
\hline Hospital stay<16 days & 137 & 90.1 \\
\hline No complications & 109 & 71.7 \\
\hline Textbook outcome & 96 & 63.1 \\
\hline
\end{tabular}

Table 3 shows the results of a logistic regression analysis identifying risk factors reducing the chance for a "textbook outcome". The logistic regression analysis, including stage of disease, pulmonary co-morbidity, smoking status, lung function and type of resection, showed that stage of disease was an independent risk factor that reduced the chance of a "textbook outcome"(OR $0.56,95 \% \mathrm{Cl}$ 0.33-0.92, $p=0.024)$. Resection type showed a trend towards significance, reducing the chance of a "textbook outcome" with OR 0.62 (95\% Confidence Interval 0.36-1.01, $p=0.066$ ). 
Table 3. Logistic regression analysis identifying risk factors reducing the chance of a "textbook outcome"

\begin{tabular}{|c|c|c|c|}
\hline Variables & $\begin{array}{l}\text { Odds } \\
\text { ratio }\end{array}$ & $95 \% \mathrm{Cl}$ & $\begin{array}{c}P \\
\text { value }\end{array}$ \\
\hline Pulmonary co-morbidity ${ }^{a}$ & & & 0.27 \\
\hline No (reference) & 1 & & \\
\hline Yes & 0.61 & $0.26-1.46$ & \\
\hline Smoking Status & & & 0.18 \\
\hline Current/former (reference) & 1 & & \\
\hline Never & 5.94 & $0.36-1.01$ & \\
\hline Lung function-FEV $1^{\mathrm{D}}$ & 1.01 & $0.13-1.02$ & 0.16 \\
\hline Resection type & & & 0.07 \\
\hline Wedge & 2.56 & $0.98-7.56$ & \\
\hline (bi/tri-)segmentectomy & 1.61 & $0.99-2.75$ & \\
\hline Lobectomy (reference) & 1 & & \\
\hline Bilobectomy/sleeve resection & 0.63 & $0.36-1.01$ & \\
\hline Pneumonectomy & 0.39 & $0.13-1.02$ & \\
\hline Stage $^{d}$ & & & 0.024 \\
\hline Stage 0 (carcinoma in situ) & 1.79 & $1.09-3.02$ & \\
\hline Stage 1 (reference) & 1 & & \\
\hline Stage 2 & 0.56 & $0.33-0.92$ & \\
\hline Stage 3 & 0.31 & $0.11-0.84$ & \\
\hline
\end{tabular}

Legend

a pulmonary co-morbidity included asthma, lung fibrosis and lung disease due to smoking.

${ }^{b}$ FEV1=forced expiratory volume in one second, volume that can be expired in one second.

${ }^{\mathrm{C}}$ Resection type was treated as a numeric factor ordering resection from the smallest resection (wedge) to the largest (pneumonectomy).

$\mathrm{d}$ Stages were treated as a numeric factor ordering stage from low (stage 0) to high stage (stage 3). 
Selecting patients who completed the quality of life questionnaire 6 months (or 3 months, when 6 month data was missing) after the operation, 72 patients were included for analysis (47 percent).
When QoL data was added to the summarizing measure, a "textbook outcome" was observed in only 13 percent of the patients (Table 4).

Table 4. Outcome after pulmonary resection for NSCLC with QoL PCS and MCS subscales

\begin{tabular}{lcc}
\hline & \multicolumn{2}{c}{ Patients } \\
\cline { 2 - 3 } & $\mathrm{N}$ & $\%$ \\
\hline Total Patients included & 72 & 100 \\
\hline No in hospital Mortality & 72 & 100 \\
\hline Intensive Care stay<3 days & 70 & 97.2 \\
\hline Radical Resection & 68 & 94.4 \\
\hline No readmission & 67 & 93.1 \\
\hline No re-intervention & 66 & 91.7 \\
\hline Hospital stay<16 days & 66 & 91.7 \\
\hline No complications & 55 & 76.4 \\
\hline Mental Composite & 42 & 58.3 \\
Scale=>Baseline & & 27.8 \\
\hline Physical Composite & 20 & \\
Scale=>Baseline & & 12.5 \\
\hline Textbook outcome & 9 & \\
\hline
\end{tabular}




\section{Discussion}

This is the first study exploring the use of a summarising outcome measure (i.e. "textbook outcome") for lung cancer surgery. A "textbook outcome" was observed in almost two thirds of the patients. When QoL data was added to the summarizing measure, a "textbook outcome" was observed in only 13 percent of the patients Stage was identified as an independent risk factor reducing the chance for a "textbook outcome".

Because this is the first study exploring "textbook outcome" for lung cancer surgery, comparing our results is difficult. Brunelli et al. developed a riskadjusted index combining four different outcomes to evaluate the quality of care for lung cancer surgery in their thoracic surgery unit [4]. Focussing on four "undesired" outcome measures, instead of "preferred outcome", the four measures they used were 30 days or in-hospital mortality, cardiopulmonary morbidity, unplanned/emergency intensive care unit admission, and prolonged length of stay (more than 14 days). This riskadjusted index seemed more comprehensive compared to individual outcomes measures: when only riskadjusted mortality was used to evaluate performance, 2007 resulted as their best year. However, when quality of care was measured using the risk-adjusted index, 2007 was ranged third. Their study presents a methodological template for developing a risk-adjusted index trying to overcome inherent limitations of individual outcomes measures. In 2008, The European Society for Thoracic Surgeons (ESTS) developed a composite measure incorporating process and outcome measures for major lung resection, to be able to rank and compare hospitals [3]. Their composite measure contained three quality domains (i.e. preoperative care, operative care and postoperative care) and provided a methodological template for performance monitoring. This composite measure aimed to measure the overall quality of surgical lung cancer care, as our study focussed on measuring the quality of postoperative care. Kolfschoten et al. focused on "textbook outcome" for colorectal cancer surgery in the Netherlands [12]. A "textbook outcome" rate of 49 percent was found. Based on this study it was concluded that this approach for measuring surgical quality can identify positive and negative outliners. In other surgical fields such as surgery for coronary artery bypass, aortic valve replacement, percutaneous coronary interventions, pancreatic cancer resection and oesophageal cancer resection, composite measures have been proposed $[8,17]$.

We explored the number and proportion of patients with a desired health care outcome (i.e. "textbook outcome") and identified risk factors reducing the chance of a "textbook outcome". Our study showed a "textbook outcome" was achieved in almost two third of the cases and was an easy to use outcome measure. This approach has a substantial event rate (in 63\% a "textbook outcome" was observed), providing opportunities to compare hospital performances with sufficient statistical power. In addition, we showed that "textbook outcome" is influenced by "case mix-factors", like tumour stage and will require a riskadjustment when it is used for hospital to hospital comparisons. Our study shows a "textbook outcome" could become an additional informative outcome indicator for lung cancer surgery because it has several advantages over individual quality of 
care indicators. It is known, that most patients prefer a summary measure on quality of care over more detailed measures when choosing a hospital for their surgery [9]. A frequently asked question at the end of a pre-operative consult is "doctor, what are the risks of this operation?"

Most patients, and surgeons, consider patient outcomes the "bottom line" of surgical practice. Therefore, "textbook outcome" could provide an easy to understand overview for patients and an easy to explain outcome measure for surgeons. Comparing individual hospitals on basis of individual outcome indicators could be difficult because a hospital may score high on one quality indicator and low on another. Therefore, a summarising measure such as we presented, could give an additional overview of quality of care. It also corresponds to the major goals of cancer surgery: to accomplish a radical resection with as little morbidity as possible. Singular outcome indicators like complication and postoperative mortality rates focus on "undesired" outcomes, which may overshadow the "desired" outcomes of cancer surgery: achieving a radical resection which improves patients' chances for long term survival.

Our study has several limitations. First, this study is a non-randomized, single centre study with a limited patient number. To validate this quality of care measure, multi-centre data is necessary, so individual hospitals can compare their "textbook outcome" rate to a population set benchmark and correct their data for case mix. More data is also necessary to identify more risk factors influencing the chance for a "textbook outcome" and improving quality if care. By comparing individual hospitals to a population-based benchmark, significant positive and negative outliers can be identified. A focus on "best practices" could provide information valuable for others to improve pre-operative evaluation, surgical treatment as well as perioperative care for their patients.

Further improvements would be to allocate each individual outcome measure its one weight; surely a longer hospital stay is a minor problem compared to postoperative mortality. Also, a clear cut off score has to be defined, to differentiate good from bad performers. These requirements are only met when multicentre data is available. Recently the Dutch Lung Surgery Audit was started, collecting detailed data on almost every patient undergoing a thoracic procedure for NSCLC. The multicentre data from this audit will allow it to validate our summary measure and compare hospital performances on "textbook outcome" in the Netherlands. Our study provides a solid foundation on which to base future research and when the Dutch Lung Surgery Audit will contribute its population-based data to the database of the European Society of Thoracic Surgeons, comparisons between hospitals in different European countries will be possible. 


\section{References}

1. Akcali Y, Demir H, Tezcan B. The effect of standard posterolateral versus muscle-sparing thoracotomy on multiple parameters. Ann Thorac Surg. 2003;76:1050-54

2. Birkmeyer JD, Dimick JB and NJO Birkmeyer. Measuring the Quality of Surgical Care: Structure, process, or Outcome? J Am Coll Surg. 2004;198:626-32.

3. Brunelli A, Berrisford RG, Rocco G et al. The European Thoracic Database project: composite performance score to measure quality of care after major lung resection. Eur J Cardiothorac Surg. 2009;35:769-74

4. Brunelli A, Refai M, Salati M, Pompili C, Sabbatini A. Standardized Combined Outcome Index as an Instrument for Monitoring Performance After Pulmonary Resection. Ann Thorac Surg. 2011;92:272-77

5. Cao C, Zhu ZH, Yan TD. Videoassisted thoracic surgery versus open thoracotomy for non-small-cell lung cancer: a propensity score analysis based on a multi-institutional registry. Eur J Cardiothorac Surg. 2013;44:849-54

6. Chen FF, Zhang D, Wang YL et al. Video-assisted thoracoscopic surgery lobectomy versus open lobectomy in patients with clinical stage non-small cell lung cancer: a meta-analysis. Eur J Surg Oncol. 2013;39:957-63

7. Dimick JB, Welch $\mathrm{HG}$ and Birkmeyer JD. Surgical Mortality as an Indicator of Hospital Quality. JAMA. 2004;294:847-51

8. Dimick JB, Staiger DO, Osborne NH et al. Composite measures for rating hospital quality with major surgery.
Health Serv Res. 2012;47:1861-79

9. Dijs-Elsinga J, Otten W, Versluijs MM et al. Choosing a hospital for surgery: the importance of information on quality of care. Med Decis Making. 2010;30:544-55

10. Fischer B, Lassen U, Mortensen J et al. Preoperative staging of lung cancer with combined PET-CT. N Engl J Med. 2009;361:32-39

11. Khan IH, McManus KG, McCraith A, McGuigan JA. Muscle sparing thoracotomy: a biomechanical analysis confirms preservation of muscle strength but no improvement in wound discomfort. Eur J Cardiothorac Surg. 2000;18:656-61

12. Kolfschoten NE, Kievit J, Gooiker GA et al. Focusing on desired outcomes of care after colon cancer resections; hospital variations in 'textbook outcome'. Eur J Surg Oncol. 2013;39:156-63

13. Kozower BD, Stukenborg GJ. Lung cancer resection volume: is procedure volume really an indicator of quality? Semin Thorac Cardiovasc Surg. 2012;24:93-98

14. Kozower BD, Stukenborg GJ. The relationship between hospital lung cancer resection volume and patient mortality risk. Ann Surg. 2011;254:1032-37

15. McHorney CA, Ware JE Jr, Lu JF et al. The MOS 36-item Short-Form Health Survey (SF-36): III. Tests of data quality, scaling assumptions, and reliability across diverse patient groups. Med Care. 1994;32:40-66

16. Numan RC, Klomp HM, Li W et al. A clinical audit in a multidisciplinary care path for thoracic surgery: an instrument for continuous quality 
improvement. Lung Cancer. 2012;78:270-75

17. Staiger DO, Dimick JB, Baser $O$ et al. Empirically derived composite measures of surgical performance. Med Care. 2009;47:226-33

18. Von Meyenfeldt EM, Gooiker GA, van Gijn W et al. The relationship between volume or surgeon specialty and outcome in the surgical treatment of lung cancer: a systematic review and meta-analysis. J Thorac Oncol. 2012;7:1170-78

19. Website of the Integraal Kankercentrum Nederland.

http://www.cijfersoverkanker.nl/meestvoorkomende-soorten-52.html

20. Website of the Integraal Kankercentrum Nederland. http://www.cijfersoverkanker.nl/selecties /Incidentie_long_en_luchtpijpkanker/ img512b296a2fc3f

21. Website of DICA, jaarraportage 2012.

http://www.clinicalaudit.nl/jaarrapportage /\#dica_rapportage

22. Website of the European Society of thoracic surgeons.

http://www.ests.org/default.aspx 
Conflict of interest Statement

All authors declare that there is no actual or potential conflict of interest of any kind including any financial, personal or other relationships with other people or organizations that could inappropriately influence (bias) our work. 